\title{
Temporary Biliary Stent Placement for Benign Stricture with Laparoscopic-Assisted Endoscopic Stent Retrieval in a Patient Status Post Roux-en-Y Gastric Bypass: A Multidisciplinary Approach to a Complex Condition
}

\author{
Kevin Anton ${ }^{1}$ Harshit S. Khara ${ }^{2}$ Ashok B. Bhanushali ${ }^{1}$ \\ ${ }^{1}$ Division of Vascular and Interventional Radiology, Department of \\ Radiology, Geisinger Medical Center, Danville, Pennsylvania, \\ United States \\ 2 Department of Gastroenterology and Hepatology, Geisinger Medical \\ Center, Danville, Pennsylvania, United States

\begin{abstract}
Address for correspondence Kevin Anton, MD, PhD, Division of Vascular and Interventional Radiology Department of Radiology, 100 North Academy Avenue, Danville, PA 17821, United States (e-mail: kevanton22@gmail.com).
\end{abstract}

J Clin Interv Radiol ISVIR 2017;1:117-123.

\begin{abstract}
Keywords

- biliary stricture

- biliary stent

- cholangioplasty

Benign biliary strictures are multifactorial in etiology and can be difficult to treat, causing significant morbidity and mortality. In patients with prior upper gastrointestinal surgery, such as Roux-en-Y gastric bypass, options for intervention are limited by the inability of an endoscope to reach the biliary ductal system due to altered anatomy. In patients with an inaccessible ductal system by endoscopic retrograde cholangiopancreatography (ERCP), percutaneous bile duct stent placement for benign strictures has traditionally been reserved for cases of failed cholangioplasty without additional surgical options. Our case details a novel multidisciplinary management approach for the treatment of a refractory biliary stricture in a patient with Roux-en-Y gastric bypass anatomy with remnant gastrectomy. Following interventional radiology-guided retrievable biliary stent placement and subsequent remodeling, laparoscopy-assisted ERCP with stent retrieval was performed through the combined efforts of minimally invasive surgery and gastroenterology.
\end{abstract}

\section{Introduction}

Biliary ductal strictures are multifactorial and categorized based on their benign or malignant etiology. Treatment strategies for benign biliary strictures are complex and frequently require multiple interventions to achieve longterm results. In patients with prior upper gastrointestinal surgery, such as Roux-en-Y gastric bypass, options are more limited due to the difficulty of an endoscope to reach the biliary ductal system for diagnostic or therapeutic intervention. Newer techniques, including balloon-assisted enteroscopy, have allowed for access to the biliary system in surgically altered anatomy but completion of the planned intervention remains difficult. ${ }^{1,2}$ In Roux-en-Y bypass pa- tients, the technical enteroscopic success of $80 \%$ and endoscopic retrograde cholangiopancreatography (ERCP) success of $70 \%$ have been reported, but these include few cases of biliary stricture. ${ }^{3}$ In patients with an inaccessible ductal system by ERCP, permanent bile duct stent placement for benign strictures has traditionally been a last resort approach for nonsurgical candidates in which cholangioplasty has failed. Bile ducts stents will inevitably occlude, and metallic stents used to treat benign strictures have demonstrated patency rates of $25 \%$ at 36 months. ${ }^{4}$

We present a case detailing a novel approach to a patient with biliary stricture following Roux-en-Y gastric bypass with remnant gastrectomy and multiple failed attempts at serial percutaneous catheter dilatations. Following interventional 
radiology (IR)-guided percutaneous transhepatic retrievable stent placement for prolonged dilatation and remodeling, laparoscopy-assisted ERCP with stent retrieval was performed through the combined efforts of minimally invasive surgery (MIS) and gastroenterology.

\section{Case Report}

A 49-year old obese female patient with a medical history significant for hypertension, hyperlipidemia, hepatic steatosis, gastroesophageal reflux disease, and 6 pack/year smoking history had undergone Roux-en-Ygastric bypass at age 45 for morbid obesity.

Three months postoperatively following Roux-en-Y, she was diagnosed with a marginal ulcer just distal to the gastrojejunal anastomosis (-Fig. 1A-D). She continued to have ulcer-related chest and epigastric discomfort despite appropriate medical management and underwent revision of the gastrojejunostomy with truncal vagotomy and remnant gastrectomy 6 months after the initial Roux-en-Y bypass. Her postoperative course was complicated by recurrent esophagojejunal anastomotic stricture requiring incisional therapy and repeat esophagogastroduodenoscopy balloon dilatation (-Fig. 1E-H). She continued to complain of intermittent epigastric pain and nausea with meals and was found to have elevated liver enzymes ( - Table $\mathbf{1}$ ), but was otherwise doing relatively well.

A magnetic resonance imaging (MRI) of the abdomen performed to evaluate her persistent abdominal pain demon- strated hepatic steatosis without evidence of biliary stricture or obstruction. Over the following 6 months, her abdominal pain progressively worsened and a repeat MRI demonstrated mild-to-moderate intrahepatic and extrahepatic biliary ductal dilatation with narrowing of the distal common bile duct, consistent with stricture ( - Fig. 2). The etiology of her stricture was felt to be postoperative, most commonly secondary to biliary injury during cholecystectomy. ${ }^{5}$

A left-sided 10F internal-external percutaneous transhepatic biliary drainage catheter was placed by IR to decompress the biliary system ( - Fig. 3 ) due to technical limitations of obtaining endoscopic access to her distal biliary tree from surgically altered anatomy. Following biliary drainage catheter placement, the ductal system decompressed. Her liver function tests (LFTs) which were trending down after peaking at aspartate aminotransferase (AST) 948, alanine aminotransferase (ALT) 623, total bilirubin 1.9 (-Table 1), normalized and remained relatively stable with minimal increases following repeat interventions.

Over the course of the next 2 years, the patient had biliary drainage catheter exchange every 6 to 8 weeks for a total of 19 exchanges. During that time, she had serial dilation of her stenosis using balloon angioplasty, and the catheter was upsized to $14 \mathrm{~F}$ to promote remodeling.

At that time, a discussion was held between the patient, IR, gastroenterology, and MIS to propose a plan to prevent this young catheter-dependent woman from requiring bimonthly catheter exchanges for the foreseeable future. Given her surgical history of Roux-en-Y gastric bypass with
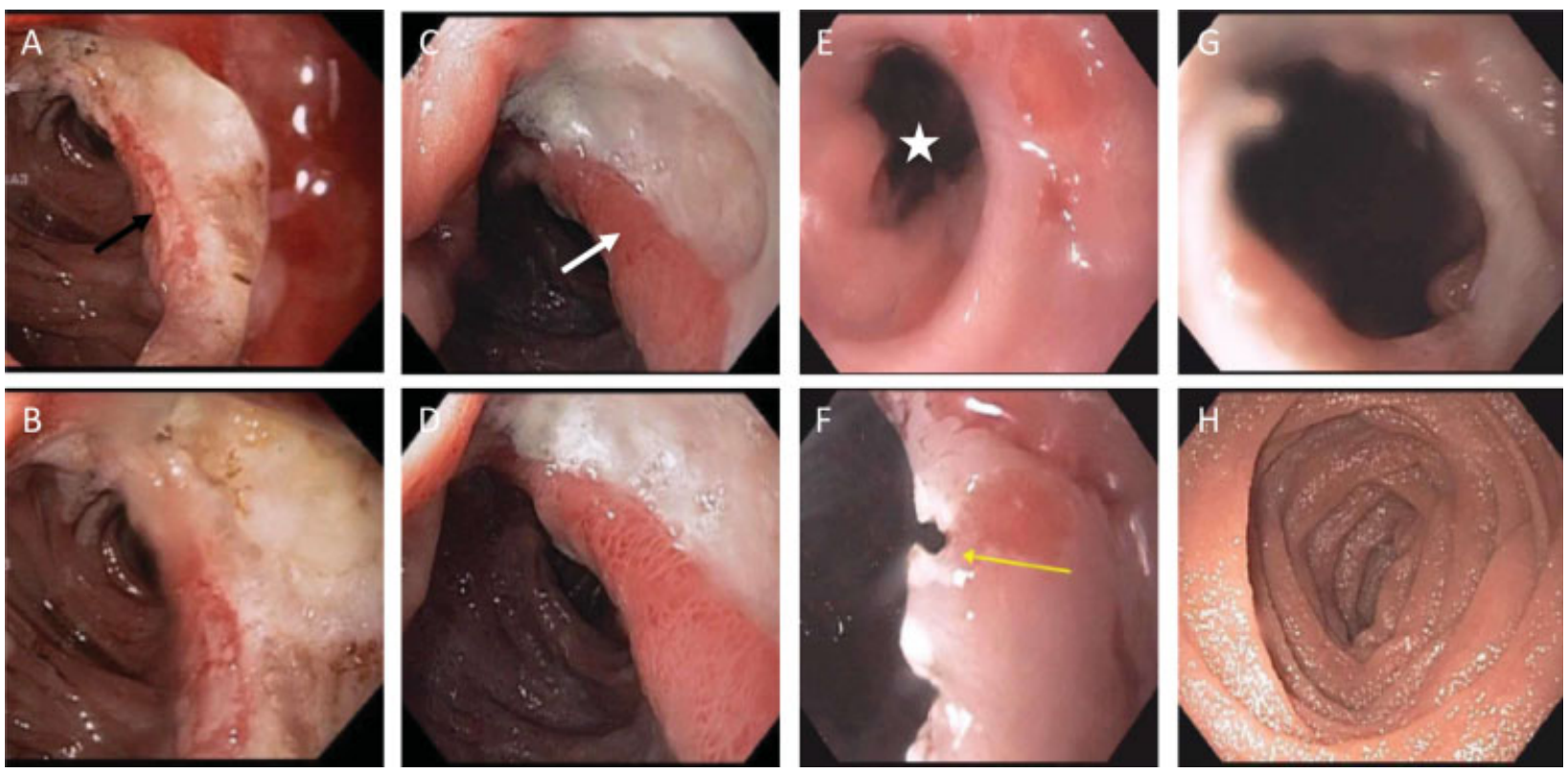

Fig. 1 Gastrojejunal (G]) anastomotic marginal ulcer status post Roux-en-Y gastric bypass. Esophagogastroduodenoscopy evaluation 3 months following Roux-en-Y gastric bypass demonstrates a large, cratered marginal ulceration just distal to the $\mathrm{G}$ J anastomosis (black arrow) measuring up to $25 \mathrm{~mm}$ in its largest dimension (A, B). Following 2 months of medical treatment, repeat endoscopic retrograde cholangiopancreatography (ERCP) demonstrated the nonbleeding cratered ulcer with clean base (white arrow) now measuring up to $15 \mathrm{~mm}$ in its largest dimension (C, D). Recurrent esophagojejunal anastomotic stricture following GJ anastomosis revision and remnant gastrectomy. EGD demonstrated a fibrotic stricture at the esophagojejunal anastomosis (white star) (E), which was treated with incisional therapy at three positions with an RX needle knife (F). After 1 month, repeat ERCP showed a benign-appearing, mild intrinsic stenosis at the esophagojejunal anastomosis (G) and a normal jejunal appearance $(\mathbf{H})$. 
Table 1 Liver enzyme values demonstrate variations with CBD stricture/obstruction with resolution following CBD stent removal

\begin{tabular}{|l|l|l|l|}
\hline & $\begin{array}{l}\text { Liver enzymes before first } \\
\text { MRI without stricture }\end{array}$ & $\begin{array}{l}\text { Liver enzymes before } \\
\text { second MRI showing } \\
\text { stricture }\end{array}$ & $\begin{array}{l}\text { 6 mo post-CBD stent } \\
\text { removal }\end{array}$ \\
\hline AST & $67(10-35 \mathrm{U} / \mathrm{L})$ & 948 & 18 \\
\hline ALT & $203(10-35 \mathrm{U} / \mathrm{L})$ & 623 & 19 \\
\hline Alkaline phosphatase & $106(0-153 \mathrm{U} / \mathrm{L})$ & 141 & 23 \\
\hline Bilirubin & $0.2(0.3-1.3 \mathrm{mg} / \mathrm{dL})$ & 1.9 & 0.2 \\
\hline Albumin & $4.6(3.8-5 \mathrm{~g} / \mathrm{dL})$ & 3.8 & 4.0 \\
\hline INR & $0.98(0.94-1.12)$ & & \\
\hline
\end{tabular}

Abbreviations: ALT, alanine aminotransferase; AST, aspartate aminotransferase; CBD, common bile duct; INR, international normalized ratio; MRI, magnetic resonance imaging.

Note: Initial laboratory values before MRI demonstrating hepatic steatosis without CBD stricture showed mildly elevated AST and ALT with normal bilirubin. At the time of repeat, MRI is demonstrating CBD stricture, AST, ALT, and bilirubin were all elevated. Following CBD stent removal, all values normalized.

remnant gastrectomy and the inability to approach the biliary system using traditional ERCP, a novel multidisciplinary approach was devised.

The patient was taken to the IR suite, and the internalexternal biliary drain was removed over a 0.035 wire.
Following appropriate measurements with a 9F marker tip sheath, a WallFlex (Boston Scientific, Marlborough, Massachusetts, United States) $10 \mathrm{~mm} \times 4 \mathrm{~cm}$ fully covered metal biliary stent was deployed percutaneously across the stricture (-Fig. 4) in an anterograde manner for stricture
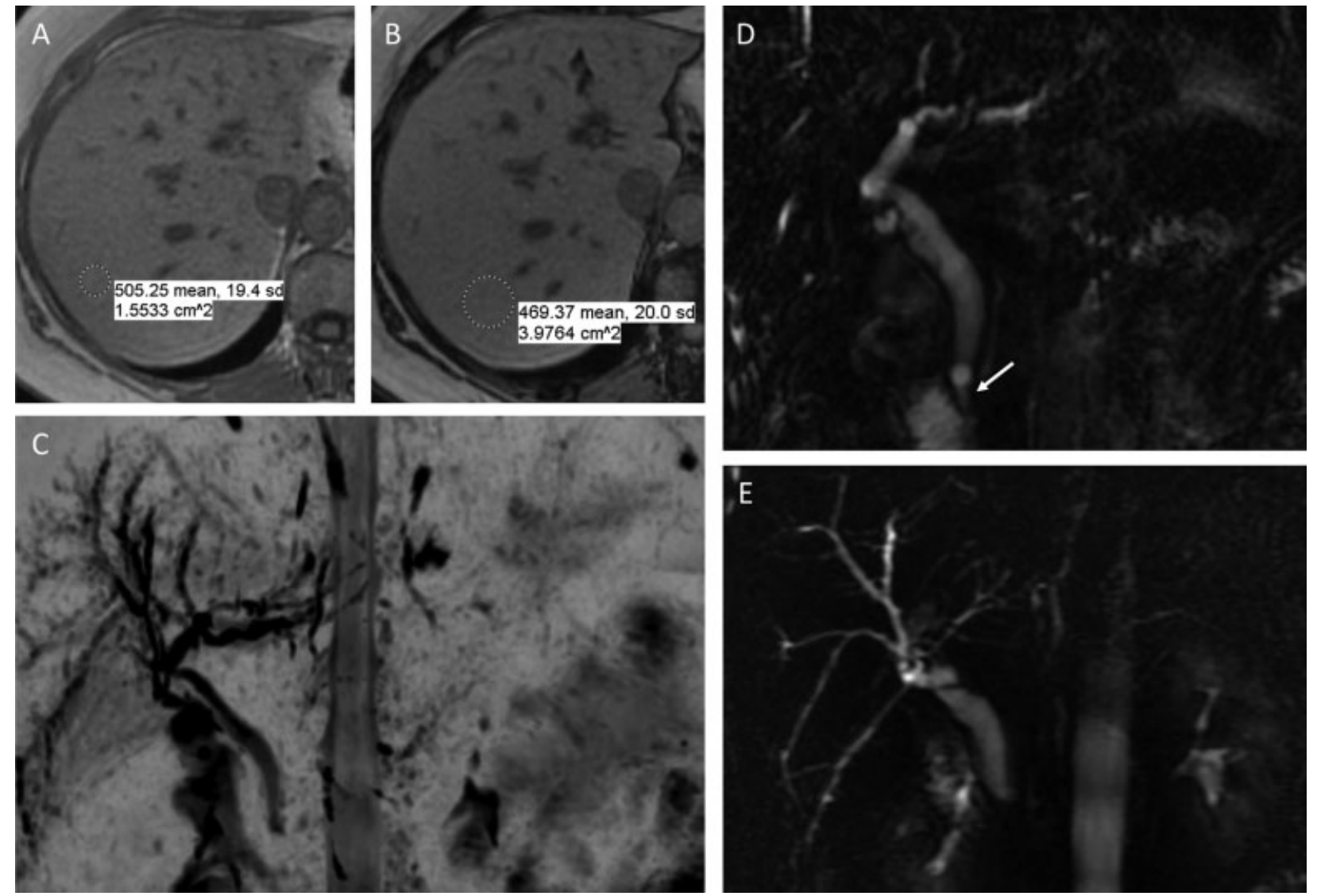

Fig. 2 Magnetic resonance imaging (MRI) evidence of hepatic steatosis and biliary duct stricture. An initial MRI with magnetic resonance cholangiopancreatography (MRCP) of the abdomen performed 21 months after the initial Roux-en-Y gastric bypass demonstrated loss of signal when analyzing in-phase (A) and out-of-phase (B) images, consistent with mild hepatic steatosis. MRCP demonstrated a normal biliary tree without evidence of obstruction (C). After 6 months, the patient experienced increasing abdominal pain and was found to have elevated liver function tests. Repeat MRCP demonstrated mild-to-moderate intrahepatic and extrahepatic biliary ductal dilatation with narrowing of the distal common bile duct (white arrow), consistent with stricture (D, E). 


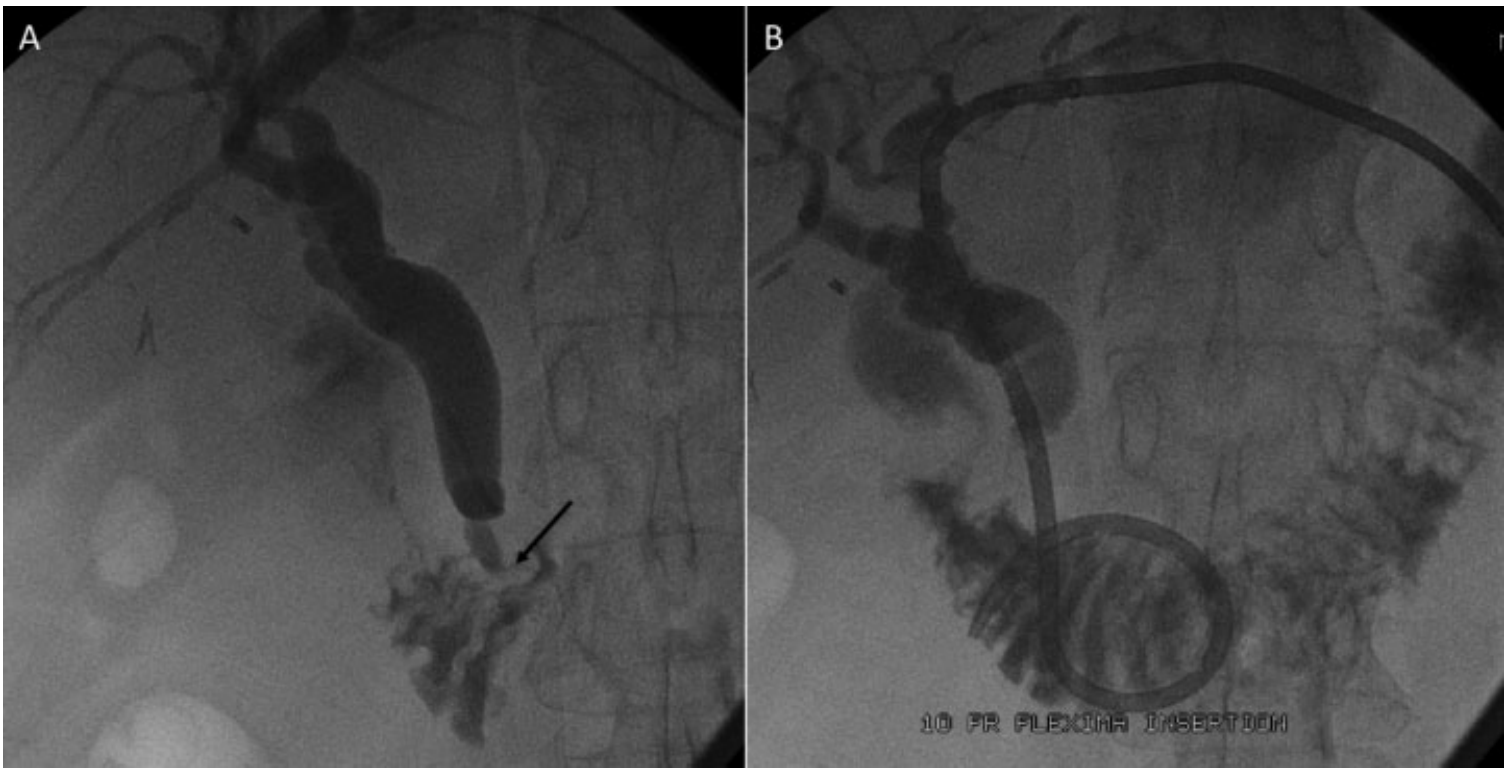

Fig. 3 Left-sided internal-external percutaneous transhepatic biliary drainage catheter placement. Cholangiogram demonstrates smooth narrowing of the common bile duct adjacent to the ampulla (black arrow), consistent with benign stricture (A). Postprocedural cholangiogram demonstrates successful placement of a left internal-external percutaneous transhepatic $10 \mathrm{~F}$ biliary drainage catheter traversing the stricture with a pigtail in the jejunum (B).
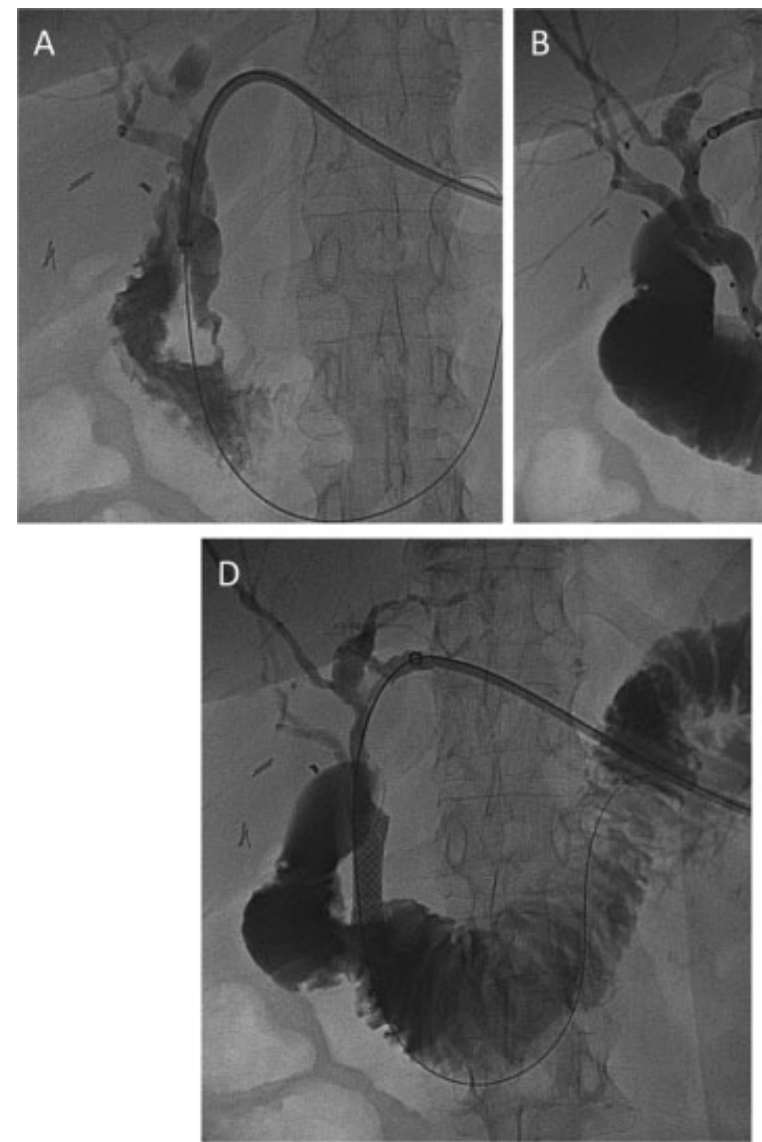
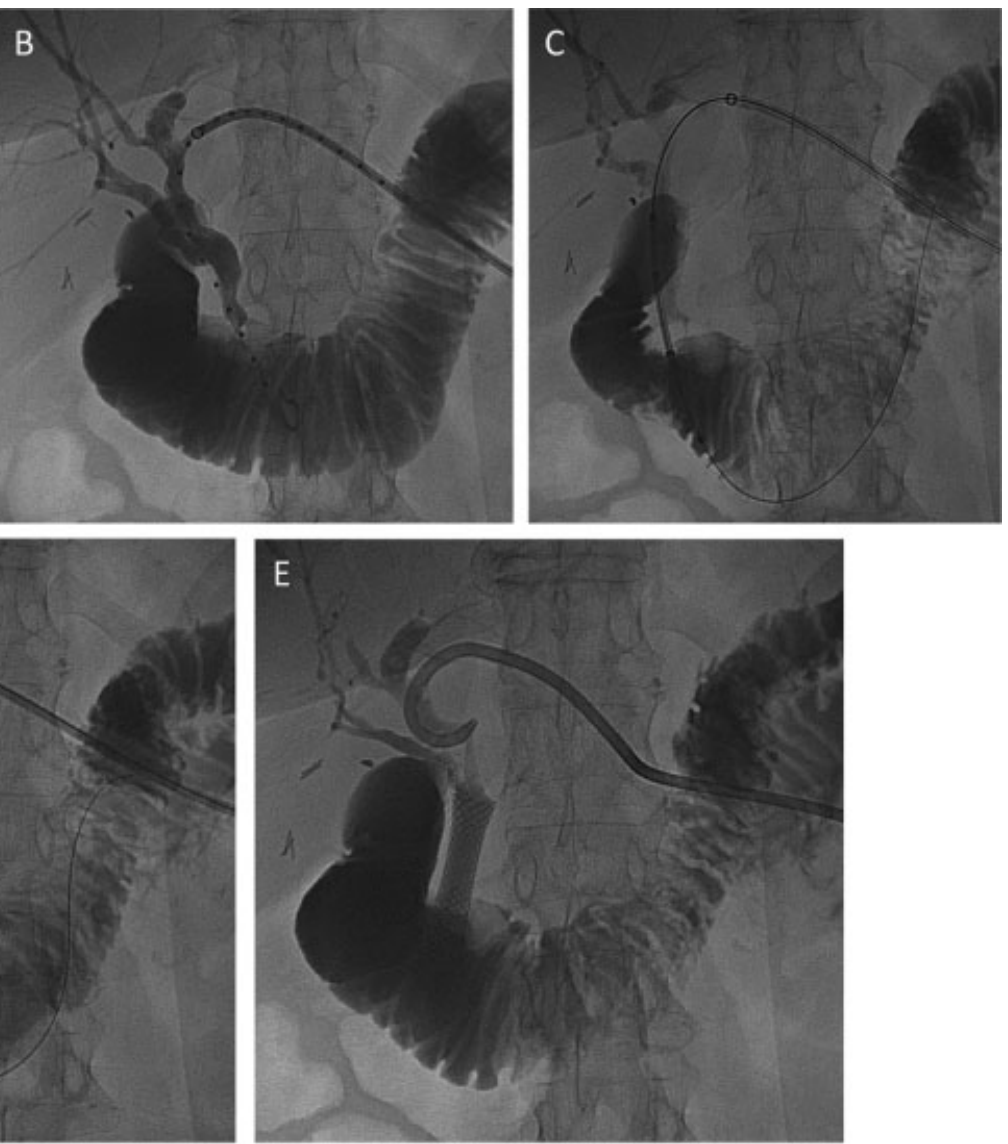

Fig. 4 Retrievable WallFlex stent (Boston Scientific) placement across the benign common bile duct stricture. Multiple fluoroscopic images demonstrate wire access across the stricture (A), stent measurement (B), and $10 \mathrm{~mm} \times 4 \mathrm{~cm}$ WallFlex stent placement (C). Cholangiogram following stent deployment demonstrates wide patency across the stricture site (D). An external drainage catheter was placed in the left biliary ductal system (E). 

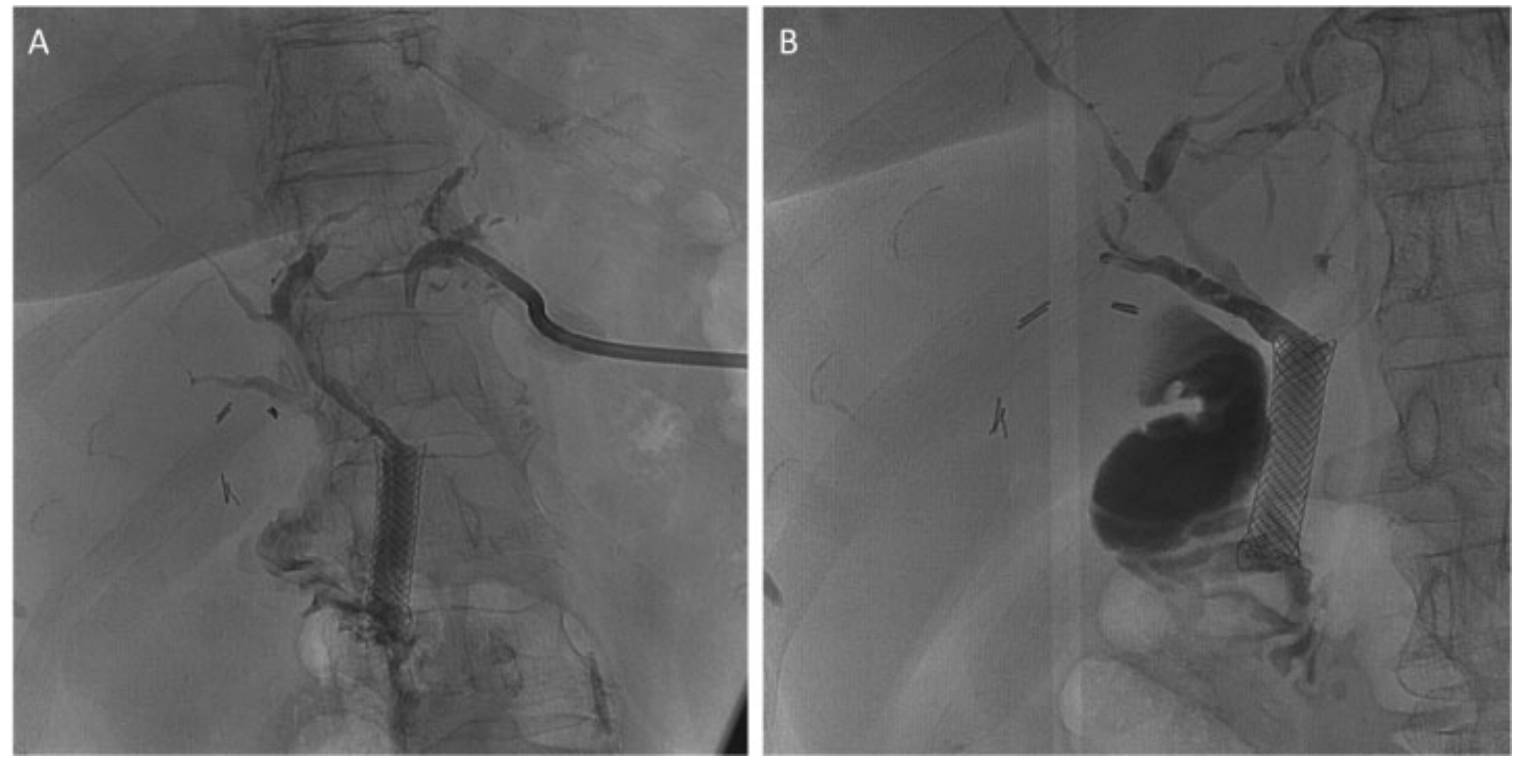

Fig. 5 Cholangiogram demonstrates short-term stent patency. Follow-up cholangiogram 2 weeks after stent placement demonstrates widely patent common bile duct stent and nondilated biliary tree (A). The external biliary drainage catheter was removed (B).

remodeling. A $12 \mathrm{~F}$ pigtail external biliary drainage catheter was placed in the left biliary system and capped after 24 hours.

After 2 weeks, a cholangiogram demonstrated widely patent biliary ducts and common bile duct stent, so the external biliary drain was removed (-Fig. 5).

Five months following initial stent placement, a successful multidisciplinary stent retrieval procedure was performed.
Since the patient has remnant gastrectomy, MIS provided laparoscopic access to the biliopancreatic (BP) limb $10 \mathrm{~cm}$ distal to the ligament of Treitz, and a $12-\mathrm{mm}$ balloon port was placed in this area. Two bowel clamps were placed distally. Gastroenterology achieved endoscopic access through the BP-limb port, and the scope was advanced in a retrograde manner to the ampullary orifice where the previously placed biliary stent was visualized in a stable position.
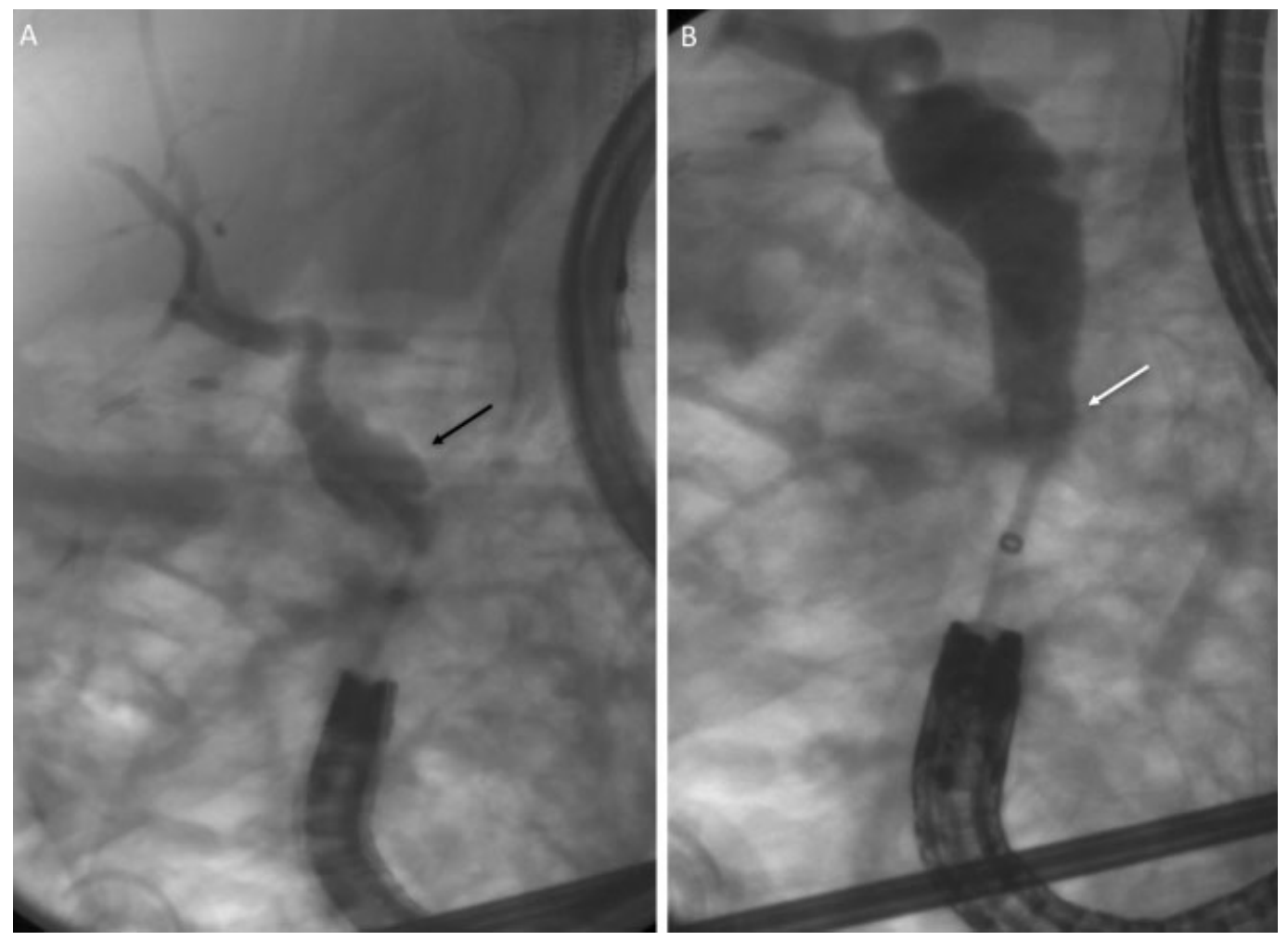

Fig. 6 Resolution of common bile duct stricture following stent removal. Endoscopic retrograde cholangiopancreatography (ERCP) immediately following laparoscopic-assisted ERCP snare stent retrieval demonstrates mildly dilated mid duct (black arrow) with smooth narrowing of the distal duct (white arrow) (A, B). The 12-mm balloon passed through the distal duct with minimal resistance, suggestive of stricture resolution. 
The stent was retrieved using a snare and found to be partially occluded using the water column test. The bile duct was then deeply cannulated using a $12-\mathrm{mm}$ balloon catheter, and a cholangiogram was obtained. The mid duct was dilated, up to $13 \mathrm{~mm}$, with a mild caliber change in the distal duct (-Fig. 6). The 12-mm balloon passed through the distal duct with minimal resistance, suggestive of stricture resolution. The ampulla was biopsied, and results were negative for a dysplastic change. The endoscope and balloon port were removed, and the enterotomy site was closed successfully.

At the time of this report, the patient is 14 months poststent retrieval with improvement in her gastrointestinal symptoms and has no symptoms or laboratory values suggestive of biliary obstruction/stricture (-Table $\mathbf{1}$ ). She continues to follow with gastroenterology for a Schatzki's ring that developed in the esophagus and has required no additional biliary intervention in that timeframe. Her most recent LFTs at 14 months poststent retrieval were AST 14 , ALT 22, and total bilirubin 0.2 .

\section{Discussion}

Common bile duct strictures can occur secondary to benign or malignant etiology. The most common causes of malignant stricture are pancreatic head adenocarcinoma and cholangiocarcinoma. The distinction between benign and malignant strictures relies on imaging and direct visualization to evaluate the morphological and associated characteristics of the stricture. Smooth, tapered margins favor benign etiology while irregular, shouldered margins and thickening and enhancement of the duct walls favors a malignant etiology. ${ }^{6}$ Diagnosis can be made by histology or cytology taken during ERCP with increasing diagnostic yield with cholangioscopically directed biopsies. $^{7-9}$

The most common causes of benign biliary strictures are iatrogenic, chronic pancreatitis, primary sclerosing cholangitis, autoimmune diseases, Mirizzi syndrome, the previous passage of stone, and ischemic cholangiopathy. ${ }^{10,11}$ Most iatrogenic cases of benign biliary stricture occur following cholecystectomy or liver transplantation. Incompletely visualized anatomy, inflammatory changes in the surgical bed, and inaccurately placed sutures and clips are likely responsible for the increase in iatrogenic bile duct injuries. ${ }^{5}$

Clinical manifestations of benign strictures vary widely from an asymptomatic presentation to findings of obstructive jaundice and cholangitis. Chronic obstruction can lead to recurrent ascending cholangitis, stone formation, liver abscess, and secondary biliary cirrhosis. ${ }^{12}$

In most cases of benign biliary stricture, ERCP has replaced surgical treatment due to its minimal invasiveness, safety profile, and the ability to perform repeat intervention. In cases of ERCP failure and patients with prior upper gastrointestinal surgery leading to a nonaccessible biliary tree, percutaneous methods can provide a successful alternative treatment. Percutaneous methods include transhepatic biliary catheter placement for decompression of an obstructed biliary system and serial dilation of the stricture. Stent placement across a bile duct stricture has shown promising results in multiple studies. ${ }^{13,14}$ In a multicenter study evaluating fully covered self-expandable metallic stents in benign extrahepatic biliary strictures, stricture resolution was demonstrated in $81 \%$ of patients after a mean of 1.2 stenting procedures with approximately 24.4 weeks before stent removal. ${ }^{15}$ The most common complications were stent migration and stent occlusion occurring in 9.7 and $4.9 \%$ of cases, respectively. This technique requires endoscopic access to the ampulla for stent placement and subsequent removal.

The American Society for Metabolic and Bariatric Surgery estimates that 179,000 bariatric surgery procedures were performed in 2013 with 34.2\% representing Roux-en-Y gastric bypass. For those patients undergoing upper gastrointestinal surgery, future development of biliary duct stricture becomes a complex problem due to anatomic variation and loss of per oral endoscopic access to the ampulla. Balloon enteroscopy-assisted ERCP is an alternative method but limits the use of traditional ERCP equipment due to its length. Lap-assisted ERCP via the gastric remnant has emerged as a successful alternative but is not feasible in patients with remnant gastrectomy, as evidenced in our patient.

Our report illustrates a novel approach allowing percutaneous access and anterograde biliary stent placement for stricture remodeling in the IR suite followed by MIS-assisted ERCP stent retrieval using a direct laparoscopic approach to the periampullary area and the biliary tree. While studies will need to evaluate the procedural efficacy and complication rates, we present one case in which the stricture was successfully treated without the need for further anatomy altering surgical resection.

\section{Conflict of Interest}

None.

Financial Disclosures

None.

\section{References}

1 Itokawa F, Itoi T, Ishii K, Sofuni A, Moriyasu F. Single- and doubleballoon enteroscopy-assisted endoscopic retrograde cholangiopancreatography in patients with Roux-en-Y plus hepaticojejunostomy anastomosis and Whipple resection. Dig Endosc 2014; 26(02, Suppl 2):136-143

2 Shah RJ, Smolkin M, Yen R, et al. A multicenter, U.S. experience of single-balloon, double-balloon, and rotational overtube-assisted enteroscopy ERCP in patients with surgically altered pancreaticobiliary anatomy (with video). Gastrointest Endosc 2013;77(04): 593-600

3 Skinner M, Popa D, Neumann H, Wilcox CM, Mönkemüller K. ERCP with the overtube-assisted enteroscopy technique: a systematic review. Endoscopy 2014;46(07):560-572

4 Gabelmann A, Hamid H, Brambs HJ, Rieber A. Metallic stents in benign biliary strictures: long-term effectiveness and interventional management of stent occlusion. AJR Am J Roentgenol 2001; 177(04):813-817 
5 Costamagna G, Boškoski I. Current treatment of benign biliary strictures. Ann Gastroenterol 2013;26(01):37-40

6 Choi SH, Han JK, Lee JM, et al. Differentiating malignant from benign common bile duct stricture with multiphasic helical CT. Radiology 2005;236(01):178-183

7 Chen YK, Parsi MA, Binmoeller KF, et al. Single-operator cholangioscopy in patients requiring evaluation of bile duct disease or therapy of biliary stones (with videos). Gastrointest Endosc 2011; 74(04):805-814

8 Meining A, Chen YK, Pleskow D, et al. Direct visualization of indeterminate pancreaticobiliary strictures with probe-based confocal laser endomicroscopy: a multicenter experience. Gastrointest Endosc 2011;74(05):961-968

9 Chathadi KV, Chandrasekhara V, Acosta RD, et al; ASGE Standards of Practice Committee. The role of ERCP in benign diseases of the biliary tract. Gastrointest Endosc 2015;81(04):795-803

10 Shanbhogue AK, Tirumani SH, Prasad SR, Fasih N, McInnes M. Benign biliary strictures: a current comprehensive clinical and imaging review. AJR Am J Roentgenol 2011;197(02):W295-306
11 Singh A, Gelrud A, Agarwal B. Biliary strictures: diagnostic considerations and approach. Gastroenterol Rep (Oxf) 2015;3(01): 22-31

12 Warshaw AL, Schapiro RH, Ferrucci JT Jr, Galdabini JJ. Persistent obstructive jaundice, cholangitis, and biliary cirrhosis due to common bile duct stenosis in chronic pancreatitis. Gastroenterology 1976;70(04):562-567

13 Haapamäki C, Kylänpää L, Udd M, et al. Randomized multicenter study of multiple plastic stents vs. covered self-expandable metallic stent in the treatment of biliary stricture in chronic pancreatitis. Endoscopy 2015;47(07):605-610

14 Devière J, Nageshwar Reddy D, Püspök A, et al; Benign Biliary Stenoses Working Group. Successful management of benign biliary strictures with fully covered self-expanding metal stents. Gastroenterology 2014;147(02):385-395, quiz e15

15 Saxena P, Diehl DL, Kumbhari V, et al. A US Multicenter Study of Safety and Efficacy of Fully Covered Self-Expandable Metallic Stents in Benign Extrahepatic Biliary Strictures. Dig Dis Sci 2015;60(11):3442-3448 